\title{
Depression and antidepressant action-from molecules to networks
}

\author{
Tomi Rantamäki ${ }^{1,2} \cdot$ Ipek Yalcin ${ }^{3}$
}

Published online: 9 May 2019

(C) Springer-Verlag GmbH Germany, part of Springer Nature 2019

\section{The great challenge}

Once in a while, we all experience feelings of hopelessness, sadness, lack of self-esteem, lack of motivation and drive, and difficulties to focus and concentrate. In far too many individuals, these physiological responses to environmental, social, or internal insults or challenges become symptoms of one of the most disabling medical conditions: the major depressive disorder (MDD). While this ancient disorder, or better said syndrome, is well recognized, the underlying causes remain poorly understood. Psychosocial stress, inflammation, persistent pain, and long-lasting sleep problems can precipitate symptoms of depression in almost all humans (and animals) but why in some people the symptoms prevail, persist, and become debilitating, even when the primary trigger wears off is unknown. Patient's emotional state is often "locked in" into melancholy, and uncontrollable self-focused rumination and negative thinking of the past, present, and future. Basic daily activities may become insurmountable leading into disability, social isolation, and further exacerbation of feelings of worthlessness, stress, and anxiety. If left untreated, major depression significantly increases the risk to develop many major chronic diseases and may lead to suicide. Unfortunately, there is a huge unmet medical need for better treatments. Commonly used prescription antidepressants often show poor efficacy and very slow onset of action and may bring numerous side

Tomi Rantamäki

tomi.rantamaki@helsinki.fi

Ipek Yalcin

yalcin@inci-cnrs.unistra.fr

1 Laboratory of Neurotherapeutics, Drug Research Program, Division of Pharmacology and Pharmacotherapy, Faculty of Pharmacy, University of Helsinki, Helsinki, Finland

2 SleepWell Research Program, Faculty of Medicine, University of Helsinki, Helsinki, Finland

3 Institut des Neurosciences Cellulaires et Intégratives, Centre National de la Recherche Scientifique and Université de Strasbourg, Strasbourg Cedex, France effects. The discovery of the rapid antidepressant effects of ketamine is a remarkable improvement in the field and the treatment is already in clinical use for the acute management of depression. However, the beneficial effects of ketamine on mood fade out almost as fast as they emerge. Ketamine may also precipitate hallucinations and is thus warranted for people with high risk of psychosis. We thus clearly need to understand the factors behind depression and the mechanisms underlying antidepressant effects much better in order to develop faster, more effective, reliable, and safe treatments that bring sustained benefit for the patients. This special issue covers some selected reviews on this important topic.

\section{It is hard to abandon monoamines}

Serendipitous discovery of monoaminergic antidepressants was a major breakthrough in psychiatry. These drugs are relatively safe and therefore widely used in inpatient and outpatient care to treat MDD and some other psychiatric and neurological illnesses. While effective for many, a significant proportion of patients are pharmacoresistant. In those who do respond, the therapeutic effects become evident with a delay of weeks or even months. There is currently no way to predict who will respond, and when, and to which of these medications.

Perez-Caballero et al. (2019) highlight strategies how to manage with some of the problems associated with the use of monoaminergic antidepressants. Unfortunately, most of such strategies are not well established and solutions must be tested in a trial-and-error basis. Roughly 20 different small chemical compounds targeting monoaminergic neurotransmission, one way or the other, are in the markets today. Some patients benefit from agents that are more selective for noradrenergic over serotonergic system, while the most unselective drugs bring best effects for some others. Curiously enough, we have witnessed renewed interest to multitarget strategies with many of the newest antidepressants. However, none of the antidepressants can be regarded as the most efficacious one. On the other hand, both the efficacy and 
onset of action of antidepressants may be improved with some "drug supplements" such as lithium, antipsychotics, or pindolol. In the most complicated cases, mixed polypharmaceutical approach is often the reality, sometimes with clear benefits. Still, many patients fail to respond to all solutions or get only marginal responses.

The glutamatergic system has been at the center of latest innovations in antidepressant field. This interest originates largely from the discovery of the rapid antidepressant effects of ketamine, an NMDA-R ( $N$-methyl-D-aspartate receptor) antagonist (Berman et al. 2000; Zanos and Gould 2018; Zarate Jr et al. 2006). Ketamine improves depression and reduces suicidal thinking very rapidly, within few hours, and the effects last for several days. An intravenous low dose of $0.5 \mathrm{mg} / \mathrm{kg}$ is commonly delivered in clinical practice. However, an intranasal esketamine has been recently approved for the treatment of MDD in the USA. The response rates to ketamine are somewhat impressive, but not all patients respond. For all those who do respond, the benefits are transient. Most patients experience relapse within a week or so. Therefore, ketamine does not provide a sole solution for the management of depression.

\section{Synaptic plasticity and antidepressant drug action: important but how it all happens?}

Accumulating evidence suggests that facilitation of intrinsic neurotrophic systems and subsequent changes in synaptic plasticity and synaptogenesis play a role in antidepressant effects while stress and depression are associated with neuronal atrophy and loss of synapses in brain areas implicated in mood regulation (Castrén et al. 2007; Duman and Aghajanian 2012). These effects of antidepressants are considered to increase the structural variability and functional plasticity in the brain that allows rewiring of neuronal connections through the guidance of environmental stimuli (Castrén and Hen 2013; Castrén and Rantamäki 2010; Rantamäki and Yalcin 2016). While such an idea is not yet unequivocally proven, it is supported by experimental evidence and clinical experience. Most importantly, this hypothesis provides rational basis why combination of psychotherapy and antidepressant is more effective than either alone.

Increased synthesis of the neurotrophin BDNF (brainderived neurotrophic factor) is considered as a critical molecular determinant underlying antidepressant-induced synaptic plasticity (Castrén et al. 2007). Essentially, all treatments of depression have been shown to upregulate BDNF synthesis in the cortex and hippocampus in animal models. BDNF levels are reduced in the cortex, hippocampus, and blood of depressed patients while antidepressants tend to normalize these changes. Moreover, the behavioral effects of antidepressants are blunted in BDNF-deficient animals and direct application of BDNF in the cortex and hippocampus produces antidepressant-like responses. Regulation of BDNF and its signaling mechanisms are, however, much more complicated than originally thought. The synthesis and processing of BDNF give rise to several mediators of synaptic plasticity, although most of the studies have focused on mature BDNF that preferentially acts on TrkB receptor. Precursor of BDNF, the pro-BDNF and the pro-peptide itself, appears to produce several neurobiological responses opposite to those of mature BDNF. Kojima et al. (2019) highlight the current understanding of the complexity of BDNF processing and present some of the latest findings linking pro-peptide in MDD. This area of study is, however, still in infancy.

While there is little doubt that antidepressants activate TrkB receptor and its downstream signaling in the adult brain (e.g., Rantamäki et al. 2007; Saarelainen et al. 2003), the precise mechanism underlying these effects is not known. Notably, both conventional and rapid-acting antidepressants activate TrkB within hours after a single treatment in rodents. This and several other obvious discrepancies are reviewed by Rantamäki (2019). Most importantly, the latest findings of his group suggest that in contrast to conventional antidepressants, rapid-acting antidepressants regulate TrkB signaling only upon drug withdrawal during a brain state dominated by the emergence of slow EEG (electroencephalogram) activity (Kohtala et al. 2018), characteristic neural oscillations of deep sleep.

The list of molecular players involved in depression phenotypes has expanded to include several neurotransmitter and neuropeptide systems, circadian genes, transcription factors, and cytokine signaling cascades, among many others. Joca et al. (2019) review the role of nitric oxide (NO), an atypical neurotransmitter, in depression and in neuronal plasticity effects evoked by antidepressants. Based on preclinical studies, inhibiting NO synthesis can lead to antidepressant-like effects through different mechanisms such as direct nNOS and/or iNOS inhibition but also indirectly through the blockade of purinergic or muscarinic receptors, through monoaminergic system or by neuroplastic mechanisms such as increased BDNF-TrkB signaling and neurogenesis. Despite the fact that the NO is an interesting target, challenges remain in developing compounds targeting specifically this molecule.

Neurogenesis is another form of adult neuroplasticity regulated by antidepressants. Indeed, it is now well established that various clinically used and experimental antidepressants such as tricyclics, SSRIs (serotonin-selective reuptake inhibitors), glutamatergic ligands, cannabinoids, tianeptine, corticotrophin-releasing factor 1 , vasopressin $1 \mathrm{~b}$ receptor antagonists, and melanin-concentrating hormone antagonist as well as electroconvulsive therapy increase the number of new hippocampal neurons (Park 2019). Neurogenic effects of antidepressants have been mostly reported in normal animals but they can also restore decreased neurogenesis induced by stress (Tanti and Belzung 2010). While some animal studies 
highlight the role of neurogenesis in antidepressant actions, the clinical importance remains unclear (Castrén and Hen 2013; Park 2019). Notably, physical exercise is among the most efficient ways to increase adult hippocampal neurogenesis in rodents.

\section{Modelling major depression in animals: translation or lost in it?}

Like in all areas of biomedicine, different animal models have been long used to model MDD and to investigate the mechanisms of action of antidepressants. Psychiatric disorders are, however, generally very difficult to model in animals. Common laboratory rodents do exhibit depression-like endophenotypes when subjected to environmental, physical, or bodily stresses that precipitate depression also in humans. Yet, many other symptoms such as depressed mood, rumination, and suicidal thinking are impossible to recapitulate in animals. As discussed by Harro (2018), we often try too hard to utilize animal models in depression/antidepressant research. Given models can be useful to assess certain endophenotypes of depression, not the full-blown syndrome. Data should be interpreted with caution and with a critical mindset. Dr. Harro also nicely points out that in neuropsychiatric research, we should not only rely on behavioral readouts. This is important to keep in mind especially when antidepressant drug actions are assessed. For example, the ability of antidepressants to facilitate synaptic plasticity is by no means restricted to disease states, or states resembling depression.

Among the core symptoms of depression, anhedonia can be relatively reliably modelled and investigated in animals. The dopaminergic neurons within the VTA (ventral tegmental area) are importantly involved in hedonic/anhedonic behavior and motivational learning. Kaufling (2019) reviews the role of VTA dopamine neurons in commonly used stress-based animal models of depression. She explains the dual role of the VTA dopamine neurons both in the development of maladaptation and adaptation leading to depressive-like phenotypes or resilience, respectively. However, the reason why the underlying dopaminergic mechanisms between environmental and social stress models are distinct even though they lead to similar symptoms is need to be studied further by using other models of depression.

One bottleneck in depression research is related to the fact that preclinical models have been largely restricted to the analysis of stress as a precipitating factor, while, for instance, chronic pain is also among the first determinants of mood disorders. Indeed, a mean prevalence rate around $50 \%$ for MDD has been reported in patients with chronic pain (Bair et al. 2003). Humo et al. (2019) review the molecular mechanisms underlying this comorbidity and ask the question whether the chronic pain and MDD share similar neural mechanisms or chronic pain modulates neural mechanisms which increase the vulnerability for depression. As highlighted in this review, the comorbidity can be explained by shared molecular mechanisms observed in both chronic pain and mood disorders such as polymorphisms in serotonin transporter (SERT, major target of SSRIs) and imbalance of inhibitory and excitatory neurotransmission or pro-inflammatory and anti-inflammatory cytokines. However, further clinical and preclinical studies are needed to examine the second hypothesis and to search for biomarkers for early diagnosis or for developing new treatment strategies.

Sleep and circadian rhythms represent another important, yet often neglected, factors in antidepressant research in murine models. Problems in sleep and altered sleep architecture are highly common during depressed states (Nutt et al. 2008). Dysregulation of the molecular clock has also been reported in depression while several antidepressants, most notably sleep deprivation and ketamine, regulate several components of circadian system. Mendoza and Vanotti (2019) present in their review current evidences that implicate the clock gene alterations as an important factor in the development of mood disorders and their treatment. They go beyond suprachiasmatic nucleus and discuss potential involvement of also other brain clocks such as epithalamic habenula and anterior cingulate cortex. Authors highlight the necessity of deciphering the interaction between genetic (chronotypes) and environmental factors (circadian misalignment) participating the development of mood-related behaviors. It probably goes without saying that species-specific circadian rhythms must be taken into consideration for these efforts, although perhaps the same mindset should be used in all studies assessing antidepressant effects (most rodent species are nocturnal).

Funding information This work has been supported by the Academy of Finland (TR; grants 276333, 305195, 312664), the Centre National de la Recherche Scientifique (IY; contract UPR3212), the University of Strasbourg (IY), the Neurex Doctorate fellow (IY), NARSAD Young Investigator Grant from the Brain \& Behavior Research Foundation (IY, 24736), French National Research Agency (ANR) through the Programme d'Investissement d'Avenir under the contract ANR-17-EURE-0022 and ANR-18-CE37-0004-01, and Fondation de France (IY; 00081244).

\section{References}

Bair MJ, Robinson RL, Katon W, Kroenke K (2003) Depression and pain comorbidity: a literature review. Arch Intern Med 163:2433-2445. https://doi.org/10.1001/archinte.163.20.2433

Berman RM, Cappiello A, Anand A, Oren DA, Heninger GR, Charney DS, Krystal JH (2000) Antidepressant effects of ketamine in depressed patients. Biol Psychiatry 47:351-354

Castrén E, Hen R (2013) Neuronal plasticity and antidepressant actions. Trends Neurosci 36:259-267. https://doi.org/10.1016/j.tins.2012.12.010

Castrén E, Rantamäki T (2010) The role of BDNF and its receptors in depression and antidepressant drug action: reactivation of 
developmental plasticity. Dev Neurobiol 70:289-297. https://oi. org/10.1002/dneu.20758

Castrén E, Võikar V, Rantamäki T (2007) Role of neurotrophic factors in depression. Curr Opin Pharmacol 7:18-21. https://doi.org/10.1016/ j.coph.2006.08.009

Duman RS, Aghajanian GK (2012) Synaptic dysfunction in depression: potential therapeutic targets. Science 338:68-72. https://doi.org/10. $1126 /$ science. 1222939

Harro J (2018) Animal models of depression: pros and cons. Cell Tissue Res. https://doi.org/10.1007/s00441-018-2973-0

Humo M, Lu H, Yalcin I (2019) The molecular neurobiology of chronic pain-induced depression. Cell Tissue Res. https://doi.org/10.1007/ s00441-019-03003-Z

Joca SRL, Sartim AG, Roncalho AL, Diniz CFA, Wegener G (2019) Nitric oxide signalling and antidepressant action revisited. Cell Tissue Res. https://doi.org/10.1007/s00441-018-02987-4

Kaufling J (2019) Alterations and adaptation of ventral tegmental area dopaminergic neurons in animal models of depression. Cell Tissue Res. https://doi.org/10.1007/s00441-019-03007-9

Kohtala S, Theilmann W, Rosenholm M, Penna L, Karabulut G, Uusitalo S, Järventausta K, Yli-Hankala A, Yalcin I, Matsui N, Wigren H-K, Rantamäki T (2018) Cortical excitability and activation of TrkB signaling during rebound slow oscillations are critical for rapid antidepressant responses. Mol Neurobiol. https://doi.org/10.1007/s12035-018-1364-6

Kojima M, Matsu K, Mizui T (2019) BDNF pro-peptide: physiological mechanisms and implications for depression. Cell Tissue Res. https://doi.org/10.1007/s00441-019-03034-6

Mendoza J, Vanotti G (2019) Circadian neurogenetics of mood disorders. Cell Tissue Res. https://doi.org/10.1007/s00441-019-03033-7

Nutt D, Wilson S, Paterson L (2008) Sleep disorders as core symptoms of depression. Dialogues Clin Neurosci 10:329-336

Park S-C (2019) Neurogenesis and antidepressant action. Cell Tissue Res. https://doi.org/10.1007/s00441-019-03043-5
Perez-Caballero L, Torres-Sanchez S, Romero-López-Alberca C, GonzálezSaiz F, Mico JA, Berrocoso E (2019) Monoaminergic system and depression. Cell Tissue Res. https://doi.org/10.1007/s00441-018-2978-8

Rantamäki T (2019) TrkB neurotrophin receptor at the core of antidepressant effects, but how? Cell Tissue Res. https://doi.org/10.1007/ s00441-018-02985-6

Rantamäki T, Yalcin I (2016) Antidepressant drug action - from rapid changes on network function to network rewiring. Prog NeuroPsychopharmacol Biol Psychiatry 64:285-292. https://doi.org/10. 1016/j.pnpbp.2015.06.001

Rantamäki T, Hendolin P, Kankaanpää A, Mijatovic J, Piepponen P, Domenici E, Chao MV, Männistö PT, Castrén E (2007) Pharmacologically diverse antidepressants rapidly activate brainderived neurotrophic factor receptor TrkB and induce phospholipaseCgamma signaling pathways in mouse brain. Neuropsychopharmacol. 32:2152-2162. https://doi.org/10.1038/sj.npp.1301345

Saarelainen T, Hendolin P, Lucas G, Koponen E, Sairanen M, MacDonald E, Agerman K, Haapasalo A, Nawa H, Aloyz R, Ernfors P, Castrén E (2003) Activation of the TrkB neurotrophin receptor is induced by antidepressant drugs and is required for antidepressant-induced behavioral effects. J Neurosci 23:349-357

Tanti A, Belzung C (2010) Open questions in current models of antidepressant action. Br J Pharmacol 159:1187-1200. https://doi.org/10. 1111/j.1476-5381.2009.00585.x

Zanos P, Gould TD (2018) Mechanisms of ketamine action as an antidepressant. Mol Psychiatry 23:801-811. https://doi.org/10.1038/mp.2017.255

Zarate CA Jr, Singh JB, Carlson PJ, Brutsche NE, Ameli R, Luckenbaugh DA, Charney DS, Manji HK (2006) A randomized trial of an N-methylD-aspartate antagonist in treatment-resistant major depression. Arch Gen Psychiatry 63:856-864. https://doi.org/10.1001/archpsyc.63.8.856

Publisher's note Springer Nature remains neutral with regard to jurisdictional claims in published maps and institutional affiliations. 